Archive for

Organic Chemistry
Arkivoc 2018, part v, 0-0

to be inserted by editorial office

\title{
A simple and efficient method for the synthesis of 4-tosyloxazoles from tosylmethyl isocyanide with $\alpha$-ketoimidoyl chlorides
}

\author{
Mahdiyeh Talebizadeh, ${ }^{\text {a }}$ Ali Darehkordi, ${ }^{\text {* }}$ and Mohammad Anary-Abbasinejad ${ }^{\mathrm{a}}$ \\ ${ }^{a}$ Department of Chemistry, Faculty of Science, Vali-e-Asr University of Rafsanjan, Rafsanjan 77176, Iran \\ E-mail: darehkordi@vru.ac.ir adarehkordi@yahoo.com
}

Received 03-07-2018

Accepted 05-21-2018

Published on line $06-17-2018$

\section{Abstract}

An efficient one-pot method for the synthesis of potentially biologically active 4-tosyloxazole derivatives is described via reaction of imidoyl chloride adducts generated in situ from the reaction of isocyanide and acyl chlorides with $p$-toluenesulfonylmethyl isocyanide (TosMIC). This reaction was carried out in the presence of sodium hydride as a base and in THF to produce 4-tosyloxazoles in excellent yield.
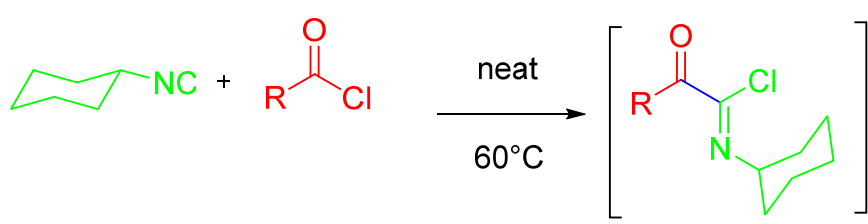

$\mathrm{R}=\mathrm{C}_{6} \mathrm{H}_{5}, 4-\mathrm{F}-\mathrm{C}_{6} \mathrm{H}_{4}$ $4-\mathrm{Br}-\mathrm{C}_{6} \mathrm{H}_{4}, 3-\mathrm{Me}-\mathrm{C}_{6} \mathrm{H}_{4}$

$3-\mathrm{Cl}-\mathrm{C}_{6} \mathrm{H}_{4}, 3-\mathrm{F}-\mathrm{C}_{6} \mathrm{H}_{4}$,

$\mathrm{ClCH}\left(\mathrm{C}\left(\mathrm{CH}_{3}\right)_{2} \mathrm{Cl}, \mathrm{CH}_{2} \mathrm{OCH}_{3}, 4-\mathrm{Me}_{-} \mathrm{C}_{6} \mathrm{H}_{4}\right.$

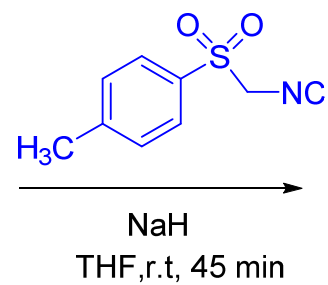

Short reaction time

Very simple procedure

Purification only with washing

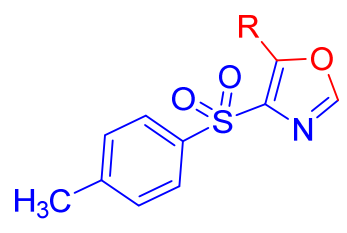

9 examples

$90-98 \%$ yield

Keywords: 4-Tosyloxazoles, $\alpha$-ketoimidoyl chloride, tosylmethyl isocyanide, oxazole 


\section{Introduction}

Oxazole compounds have attracted much attention due to their emergence as subunits of interesting biological and pharmacological compounds with anticancer ${ }^{1,2}$, antibacterial ${ }^{3,4}$, anti-inflammatory ${ }^{5,6}$ and antitubercular activities. ${ }^{7-10}$ Also, these compounds are used as valuable precursors in many useful synthetic transformations. ${ }^{11-13}$ Oxazoles are commonly synthesized using the Hantzsch reaction ${ }^{14,15}$ or by cyclodehydration of ketoamides. ${ }^{16-19}$ Synthesis via dehydration of 2-acylaminoketones is an important method for the preparation of a wide range of 2,5-disubstituted and 2,4,5-trisubstituted oxazole derivatives. ${ }^{20,21}$ Alkyl isocyanoacetates, methyl isocyanoacetate were exploited by different groups towards the synthesis of 5substituted oxazole derivatives as well as 5-(aminomethyl) oxazoles. ${ }^{22-26}$ Sasaki prepared bisoxazole derivatives which were substituted at the 4-position and dioxazolo metacyclophane derivatives using TosMIC. ${ }^{27}$ Oxazole derivatives with substitution at the 5 -position were synthesized by cyclocondensation of aldehydes with monosubstituted tosylmethyl isocyanide in moderate yield ${ }^{28-39}$ and by reaction of aryl aldehydes with TosMIC and also metalated methyl isocyanides. ${ }^{40-44}$ Although there are many methods for the synthesis of oxazoles, ${ }^{45-48} \mathrm{few}$ approaches are available for the preparation of 4,5-disubstituted oxazoles. ${ }^{28-39}$ Reaction of $\alpha$-diazo- $\beta$-keto- carboxylates and -phosphonates with arene carboxamides gives 2-aryloxazole-4carboxylates and 4-phosphonates by carbene $\mathrm{N}-\mathrm{H}$ insertion, followed by cyclodehydration results in the formation of oxazole-5-carboxylates and 5-phosphonates. ${ }^{49}$ NIS-mediated iodocyclization of $\mathrm{N}$-sulfonyl propargylamides is developed for the synthesis of various oxazolidines and iodoalkylidenedihydrooxazoles via a 5 -exo-dig process. ${ }^{46}$ According to this investigation and literature reports ${ }^{50,29}$ reactions directly arising from acid chlorides are useful and high yielding methods for synthesis of functionalized oxazoles.

\section{Results and Discussion}

A literature search revealed that only 5-phenyl-4-tosyloxazole has been synthesized from reaction of benzoyl chloride and TosMIC in the presence of $\mathrm{K}_{2} \mathrm{CO}_{3}$ as base in dimethoxyethane at $20{ }^{\circ} \mathrm{C}$, but the yield of this reaction is low $(50-55 \%)^{39}$ (Scheme 1$)$.

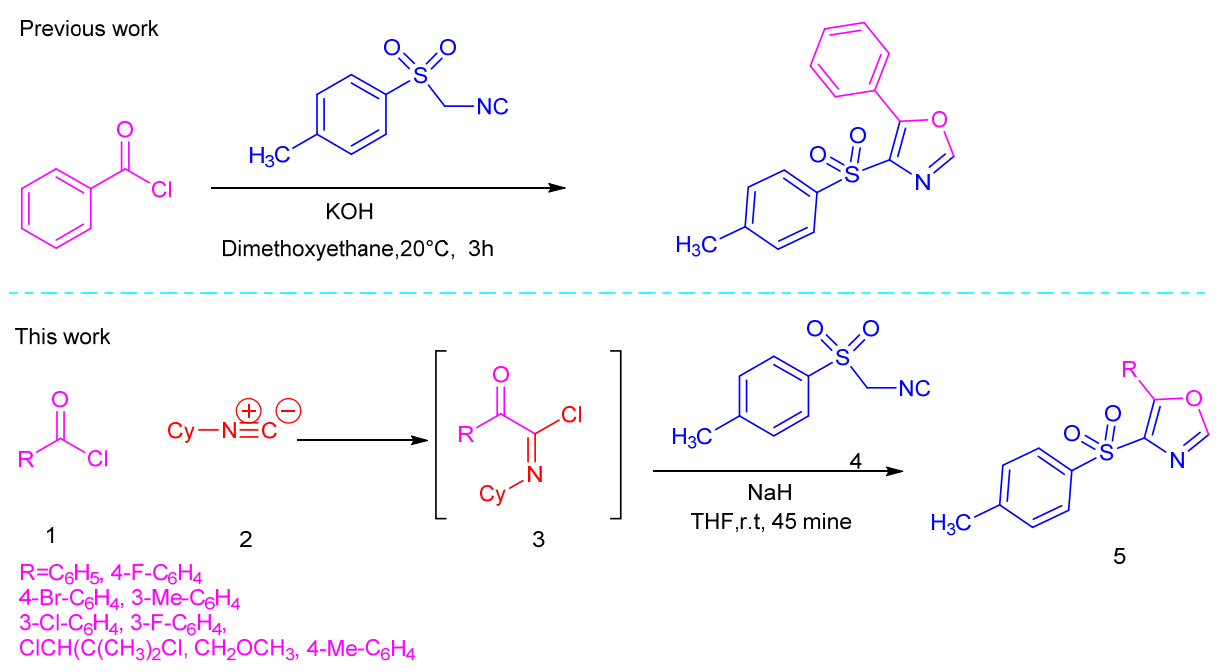

Scheme 1. Methods for the synthesis of 4-tosyloxazoles. 
Due to the importance of oxazoles in medicinal chemistry and drug discovery, and also, in continuation of our work to develop syntheses of heterocyclic compounds from imidoyl chlorides, ${ }^{48}$ we were looking to synthesize 4-tosyloxazole derivatives by condensation of aromatic and aliphatic $\alpha$-ketoimidoyl chlorides with cyclohexyl isocyanide. In this work, we report a simple method for the synthesis of 4-tosyloxazole derivatives by condensation of aromatic and aliphatic $\alpha$-ketoimidoyl chlorides with TosMIC in THF as solvent in excellent yields without using any catalyst. The Nef isocyanide adducts $\mathbf{3}$ obtained from addition of isocyanide $\mathbf{1}$ with aromatic or aliphatic acyl chloride $\mathbf{2}$ was reacted at ambient temperature with TosMIC to afford 5-phenyl-4tosyloxazole (5a, Scheme 2).

In search for selective and efficient conditions, different solvent such as THF, $\mathrm{CH}_{3} \mathrm{CN}$ and $\mathrm{CH}_{2} \mathrm{Cl}_{2}$ (at room temperature and reflux conditions) and also a variety of bases, including $\mathrm{K}_{2} \mathrm{CO}_{3}, \mathrm{NaH}$, and $\mathrm{Et}_{3} \mathrm{~N}$ were studied. As mentioned in Table 1, in the presence of $\mathrm{Et}_{3} \mathrm{~N}$ or $\mathrm{K}_{2} \mathrm{CO}_{3}$ (in THF solvent at room temperature and reflux conditions) and also in the presence of $\mathrm{K}_{2} \mathrm{CO}_{3}$ (in $\mathrm{CH}_{2} \mathrm{Cl}_{2}$ solvent at room temperature), we did not obtain any products (Table 1, entries 1-5). But when $\mathrm{NaH}$ was used as a base in $\mathrm{THF}$ or $\mathrm{CH}_{3} \mathrm{CN}$ at room temperature, the corresponding product was produced in excellent yields (entry 6-7). Therefore, all of the reactions were performed in THF in the presence of $\mathrm{NaH}$ at room temperature. To extend the scope of the reaction, the optimized protocol was applied for the synthesis of 4-tosyloxazoles by reaction with different imidoyl chlorides (Table 2). $\alpha$-Ketoimidoyl chlorides 1a-i bearing various electron-donating functional groups at various positions reacted with TosMIC (2) smoothly and the corresponding compounds (5a-i) were obtained in excellent yield (Table 2). But $\alpha$-ketoimidoyl chlorides with electron-withdrawing functional groups such as 4$\mathrm{NO}_{2}-\mathrm{C}_{6} \mathrm{H}_{4}, 3-\mathrm{NO}_{2}-\mathrm{C}_{6} \mathrm{H}_{4}$, pyridin-4-yl, $\mathrm{EtO}_{2} \mathrm{CCO}$ and $\mathrm{MeO}_{2} \mathrm{CCO}$ in the same reaction conditions, did not produce any products. The results are summarized in Table 2.

Table 1. Model reaction, conditions and yields

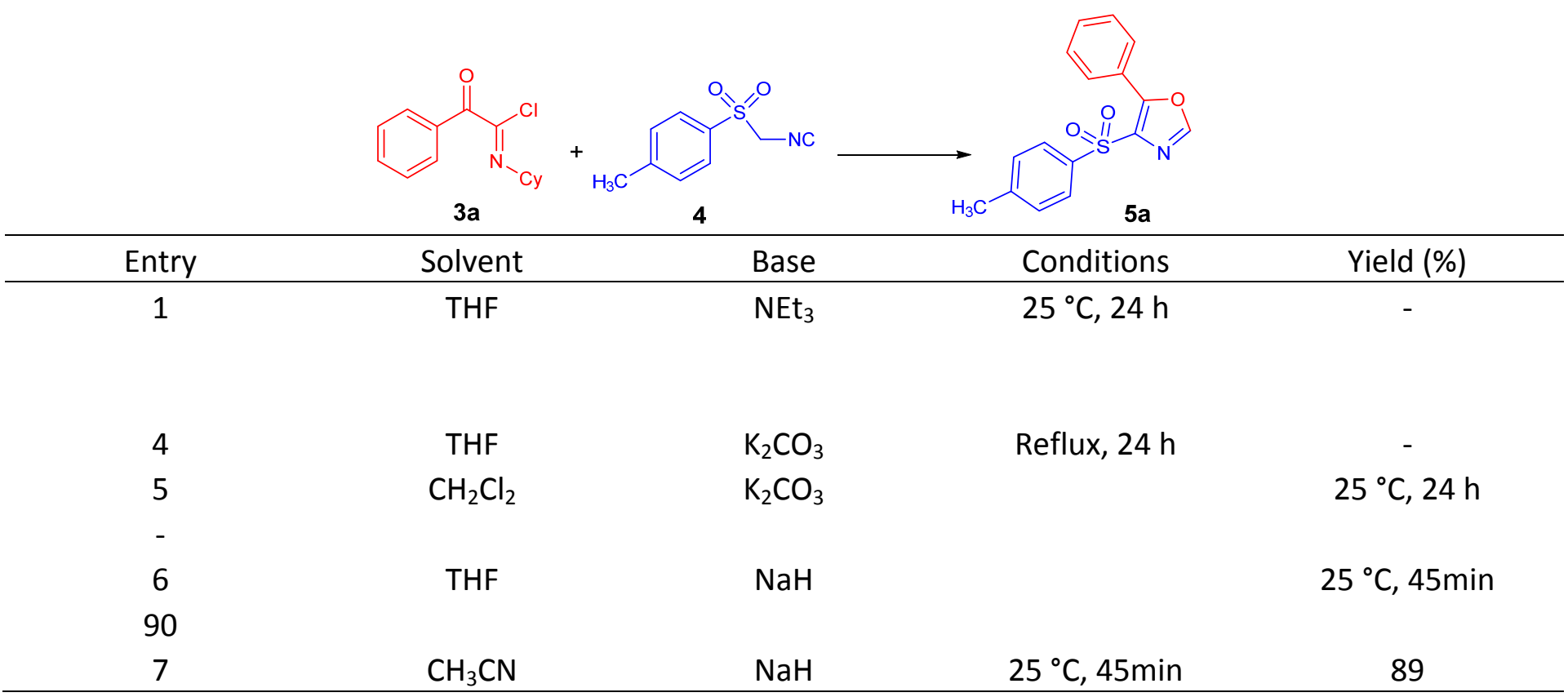


Table 2. Synthesis of 4-tosyloxazole derivatives from tosylmethyl isocyanide and $\alpha$ - ketoimidoyl chlorides
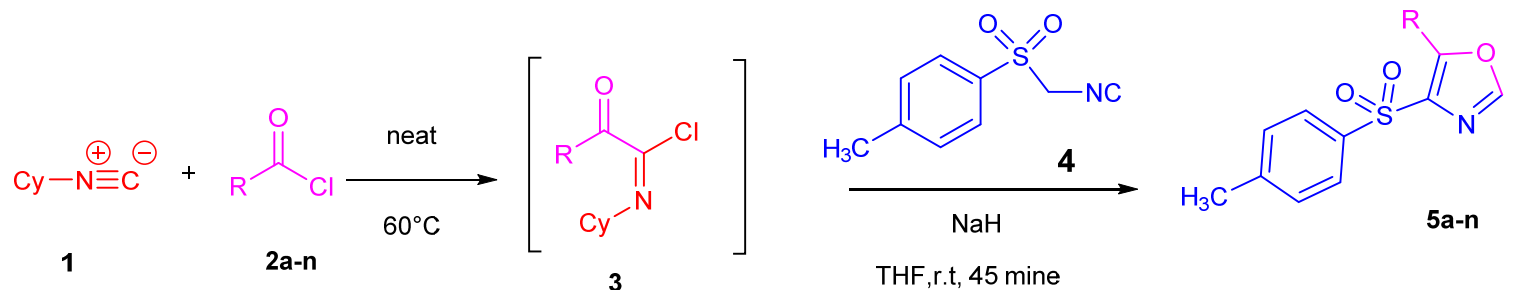

THF,r.t, 45 mine

\begin{tabular}{|c|c|c|c|c|}
\hline Entry & $\mathrm{R}$ & Product & Melting point $\left({ }^{\circ} \mathrm{C}\right)$ & Isolated Yield (\%) \\
\hline 1 & $\mathrm{C}_{6} \mathrm{H}_{5}$ & $5 a$ & $134-135$ & 90 \\
\hline 2 & $4-\mathrm{BrC}_{6} \mathrm{H}_{4}$ & $5 b$ & $153-155$ & 98 \\
\hline 3 & $4-\mathrm{FC}_{6} \mathrm{H}_{4}$ & 5c & $214-216$ & 96 \\
\hline 4 & $3-\mathrm{FC}_{6} \mathrm{H}_{4}$ & $5 d$ & $193-195$ & 96 \\
\hline 5 & $3-\mathrm{ClC}_{6} \mathrm{H}_{4}$ & $5 e$ & $260-262$ & 97 \\
\hline 6 & $4-\mathrm{MeC}_{6} \mathrm{H}_{4}$ & $5 f$ & $281-282$ & 96 \\
\hline 7 & $3-\mathrm{MeC}_{6} \mathrm{H}_{4}$ & $5 g$ & $270-272$ & 95 \\
\hline 8 & $\mathrm{CH}_{3} \mathrm{OCH}_{2}$ & $5 h$ & $200-212$ & 98 \\
\hline 9 & $\mathrm{Cl}(\mathrm{CH} 3)_{2} \mathrm{CCHCl}$ & $5 \mathbf{i}$ & $180-182$ & 98 \\
\hline 10 & $4-\mathrm{NO}_{2}-\mathrm{C}_{6} \mathrm{H}_{4}$ & $5 j$ & - & - \\
\hline 11 & $3-\mathrm{NO}_{2}-\mathrm{C}_{6} \mathrm{H}_{4}$ & $5 k$ & - & - \\
\hline 12 & pyridin-4-yl & 51 & - & - \\
\hline 13 & $\mathrm{EtO}_{2} \mathrm{CCO}$ & $5 m$ & - & - \\
\hline 14 & $\mathrm{MeO}_{2} \mathrm{CCO}$ & $5 n$ & - & - \\
\hline
\end{tabular}

A possible mechanism is proposed in Scheme 2. The formation of 4-tosyloxazole 5 by reaction of cyclohexyl isocyanide, acyl chloride and TosMIC can be rationalized by initial formation of the ketoimidoyl chloride adducts $\mathbf{3}$ by the reaction of cyclohexyl isocyanide $\mathbf{1}$ with acyl chlorides $\mathbf{2}$. Then, the intermediate $\mathbf{3}$ is attacked by TosMIC 4 to form the intermediate $\mathbf{A}$, which then undergoes an intramolecular cyclization reaction to afford product 5 (Scheme 2 ).

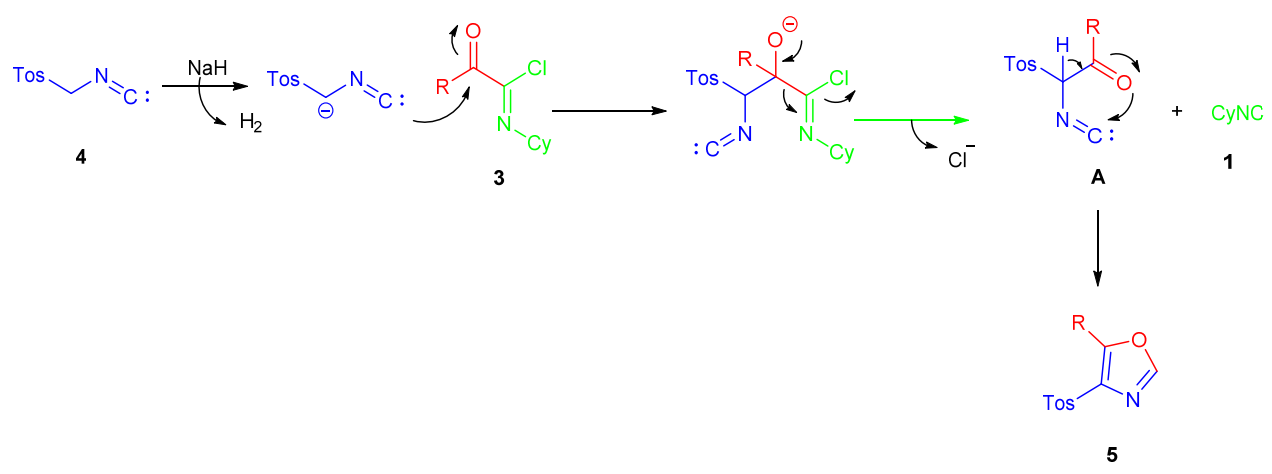

Scheme 2. Plausible mechanism for the synthesis of 4-tosyloxazole.

According to the mechanism reaction, the second step (intramolecular cyclization reaction of intermediate $\mathbf{A}$ to product $\mathbf{5}$ ) is suggested to be very important. So, the electron-donating groups on the $\alpha$ - 
ketoimidoyl chlorides increase the reaction rate and the electron-withdrawing groups on the $\alpha$-ketoimidoyl chlorides decrease the reaction rate. The structures of compounds 5a-i were deduced by the IR, NMR spectral and elemental analytical data. For example the ${ }^{1} \mathrm{H}-\mathrm{NMR}$ spectrum of 5 a exhibited a singlet at $\delta=8.90$ due to the oxazole proton. Multiplet at $\delta=7.89-7.91$, doublet at $\delta=7.84,{ }^{3} \mathrm{JHH}_{\mathrm{HH}}=8.0 \mathrm{~Hz}$, multiplet at $\delta=7.59-7.61$ and doublets at $\delta=7.46,{ }^{3} \mathrm{~J}_{\mathrm{HH}}=8.2 \mathrm{~Hz}$ correspond to nine aromatic protons present in the molecule. The signal a $\delta=$ 2.41 was assigned to the methyl protons. The ${ }^{13} \mathrm{C}-\mathrm{NMR}$ spectrum of $\mathbf{5 a}$ displayed a downfield signal at $\delta=$ 152.22 for the carbon $\mathrm{C} 2$ of the oxazole ring and at $\delta=21.60$ for methyl carbon.

\section{Conclusions}

In summary, the reaction of ketoimidoyl chlorides with TosMIC in THF in the presence of sodium hydride (NaH) as base at room temperature provides a facile and efficient route for the synthesis of 4-tosyloxazole derivatives in excellent yields. The advantages of this method are high yield, readily available starting materials, simple procedure and a straightforward purification of the products.

\section{Experimental Section}

General. ${ }^{1} \mathrm{H}-\mathrm{NMR}$ spectra were recorded in DMSO- $d_{6}$ on a Bruker model DRX-400 AVANCE spectrometer (400 $\mathrm{MHz}$ ) with TMS as internal standard ${ }^{13} \mathrm{C}-\mathrm{NMR}$ spectra were taken a by a Bruker model DRX-400 AVANCE (100 $\mathrm{MHz}$ ) spectrometer. Chemical shifts are given in ppm relative to TMS, the coupling constants J are given in $\mathrm{Hz}$. Melting points were determined on a Melt-Tem II melting point apparatus and are uncorrected. IR spectra were obtained on a Thermo scientific, Nicolet is10 FT-IR spectrometer. Peaks are reported in wave numbers $\left(\mathrm{cm}^{-1}\right)$. Element analyses (CHN) were performed with a EUROVECTOR EuroEA3000 CHNSO analyzer.

Lenochka, $d$ has to be italic (not 6). Could you please change in all Experimental Section. Thanks.

General procedure for the synthesis of compounds $5 a-i$

In a typical experimental procedure, a dry, two-necked, $50 \mathrm{~mL}$ round bottomed flask was charged with 1.0 $\mathrm{mmol}$ of acyl chloride derivatives and $1.0 \mathrm{mmol}$ of cyclohexyl isocyanide and heated at $60{ }^{\circ} \mathrm{C}$ for $1 \mathrm{~h}$. Then a mixture of TosMIC $(1.0 \mathrm{mmol})$ and sodium hydride $(60 \% \mathrm{w} / \mathrm{w})(1.0 \mathrm{mmol})$ in THF $(10 \mathrm{ml})$ was added. The mixture was stirred at room temperature for $45 \mathrm{~min}$. After completion of the reaction, as indicated by TLC (ethyl acetate : n-hexane $=1: 3$ ), the solvent was evaporated at reduced pressure; the precipitate was washed with diethyl ether $(10 \mathrm{ml})$ and was recrystallized from $95 \%$ ethanol to afford pure products $5 a-i$.

5-Phenyl-4-tosyloxazole (5a). Light yellow solid, mp $134-135^{\circ} \mathrm{C}$ (Yield: $\left.98 \%\right)$. IR ( $\left.\mathrm{KBr}\right)\left(\overline{\mathrm{U}}_{\max }, \mathrm{cm}^{-1}\right) ; 1595(\mathrm{C}=\mathrm{N})$, 1314, $1144(\mathrm{~S}=\mathrm{O}) .{ }^{1} \mathrm{H}-\mathrm{NMR}\left(400 \mathrm{MHz}, \mathrm{DMSO}-d_{6}\right): \delta(\mathrm{ppm}) 2.41\left(3 \mathrm{H}, \mathrm{s}, \mathrm{CH}_{3}\right), 7.46\left(2 \mathrm{H}, \mathrm{d},{ }^{3} \mathrm{~J}_{\mathrm{HH}} 8.1 \mathrm{~Hz}, \mathrm{HAr}\right), 7.59-$ $7.61(3 \mathrm{H}, \mathrm{m}, \mathrm{HAr}), 7.84\left(2 \mathrm{H}, \mathrm{d},{ }^{3} \mathrm{~J}_{\mathrm{HH}} 8.1 \mathrm{~Hz}, \mathrm{HAr}\right), 7.89-7.91(2 \mathrm{H}, \mathrm{m}, \mathrm{HAr}), 8.90\left(1 \mathrm{H}, \mathrm{s}\right.$, oxazole proton). ${ }^{13} \mathrm{C}-\mathrm{NMR}$ $\left(100 \mathrm{MHz}, \mathrm{DMSO}-d_{6}\right): \delta$ (ppm) $21.60\left(\mathrm{CH}_{3}\right), 125.74,125.98,128.53,128.71,129.16,129.42,130.55,131.49$, 137.19, 145.56, 152.22 (C=N). Calcd. For $\left(\mathrm{C}_{16} \mathrm{H}_{13} \mathrm{NO}_{3} \mathrm{~S}\right)$ : $\mathrm{C}, 64.18 ; \mathrm{H}, 4.39 ; \mathrm{N}, 4.69 ; \mathrm{S}, 10.70 \%$. Found: $\mathrm{C}, 64.20 ; \mathrm{H}$, $4.38 ; \mathrm{N}, 4.68 ; \mathrm{S}, 10.71 \%$.

5-(4-Bromophenyl)-4-tosyloxazole (5b). Colorless crystals, $\mathrm{mp} 153-155{ }^{\circ} \mathrm{C}$ (Yield: $\left.90 \%\right) . \mathrm{IR}(\mathrm{KBr}) \bar{U}_{\max }, \mathrm{cm}^{-1}$; $1594(\mathrm{C}=\mathrm{N}), 1332,1149(\mathrm{~S}=\mathrm{O}) .{ }^{1} \mathrm{H}-\mathrm{NMR}\left(400 \mathrm{MHz}, \mathrm{DMSO}-d_{6}\right): \delta(\mathrm{ppm}) 2.40\left(3 \mathrm{H}, \mathrm{S}, \mathrm{CH}_{3}\right), 7.47\left(2 \mathrm{H}, \mathrm{d},{ }^{3} \mathrm{JHH} 8.1 \mathrm{~Hz}\right.$, $\mathrm{HAr}), 7.83-7.85(6 \mathrm{H}, \mathrm{m}, \mathrm{HAr}) .8 .68\left(1 \mathrm{H}, \mathrm{s}\right.$, oxazole proton). ${ }^{13} \mathrm{C}-\mathrm{NMR}\left(100 \mathrm{MHz}, \mathrm{DMSO}-d_{6}\right): \delta(\mathrm{ppm}) 21.60\left(\mathrm{CH}_{3}\right)$, $124.95,125.24,128.32,130.58,131.39,132.25,135.45,136.98,145.68,151.29,152.44$ (C=N). Calcd. for $\left(\mathrm{C}_{16} \mathrm{H}_{12} \mathrm{BrNO}_{3} \mathrm{~S}\right): \mathrm{C}, 50.83 ; \mathrm{H}, 30.19 ; \mathrm{N}, 3.71 ; \mathrm{S}, 8.49 \%$. Found: $\mathrm{C}, 50.81 ; \mathrm{H}, 30.20 ; \mathrm{N}, 3.70 ; \mathrm{S}, 8.48 \%$. 
5-(4-Fluorophenyl)-4-tosyloxazole (5c). Yellow solid, mp $214-216^{\circ} \mathrm{C}$ (Yield: 98\%). IR (KBr) $\left(\bar{U}_{\max }, \mathrm{cm}^{-1}\right) ; 1609$ $(\mathrm{C}=\mathrm{N}) 1329,1149(\mathrm{~S}=\mathrm{O}) .{ }^{1} \mathrm{H}-\mathrm{NMR}\left(400 \mathrm{MHz}, \mathrm{DMSO}-d_{6}\right): \delta(\mathrm{ppm}) 2.41\left(3 \mathrm{H}, \mathrm{S}, \mathrm{CH}_{3}\right), 7.43-7.48(4 \mathrm{H}, \mathrm{m}, \mathrm{HAr}), 7.84$ $\left(2 \mathrm{H}, \mathrm{d},{ }^{3} \mathrm{~J}_{\mathrm{HH}} 8.1 \mathrm{~Hz}, \mathrm{HAr}\right), 7.96\left(2 \mathrm{H}, \mathrm{dd},{ }^{3} \mathrm{~J}_{\mathrm{HF}} 8.2 \mathrm{~Hz},{ }^{3} \mathrm{~J}_{\mathrm{HH}} 4 \mathrm{~Hz}, \mathrm{HAr}\right), 8.66\left(1 \mathrm{H}, \mathrm{s}\right.$, oxazole proton); ${ }^{13} \mathrm{C}$ NMR (100 $\left.\mathrm{MHz}, \mathrm{DMSO}-d_{6}\right): \delta(\mathrm{ppm}) 21.59\left(\mathrm{CH}_{3}\right), 116.36\left(\mathrm{~d},{ }^{2} J_{\mathrm{CF}} 22.0 \mathrm{~Hz}\right), 122.31,128.27,130.55,132.10\left(\mathrm{~d},{ }^{3} J_{\mathrm{CF}} 9.1 \mathrm{~Hz}\right)$, 134.94, 137.10, 145.60, 151.50, 152.22(C=N), 162.75( $\left.\mathrm{d}^{1} \mathrm{~J}_{\mathrm{CF}} 248 \mathrm{~Hz}\right)$ Calcd. For $\left(\mathrm{C}_{16} \mathrm{H}_{12} \mathrm{FNO}_{3} \mathrm{~S}\right): \mathrm{C}, 60.55 ; \mathrm{H}, 3.83$; N, 4.40; S, 10.12\%. Found: C, 60.56; H, 3.81; N, 4.41; S, $10.10 \%$.

5-(3-Fluorophenyl)-4-tosyloxazole (5d). Light yellow solid, mp 193-195 ${ }^{\circ} \mathrm{C}$ (Yield: $\left.98 \%\right) . \mathrm{IR}(\mathrm{KBr})\left(\overline{\mathrm{U}}_{\max }, \mathrm{cm}^{-1}\right)$; $1590(\mathrm{C}=\mathrm{N})$ 1330, 1150 (S=O). ${ }^{1} \mathrm{H}-\mathrm{NMR}\left(400 \mathrm{MHz}, \mathrm{DMSO}-d_{6}\right): \delta(\mathrm{ppm})$ 2.41(3H, S, $\left.\mathrm{CH}_{3}\right), 7.47-7.48(3 \mathrm{H}, \mathrm{m}, \mathrm{HAr})$, $7.66\left(1 \mathrm{H}, \mathrm{dd},{ }^{3} J_{\mathrm{HF}} 12.1 \mathrm{~Hz},{ }^{3} \mathrm{~J}_{\mathrm{HH}} 8.0 \mathrm{~Hz}, \mathrm{HAr}\right), 7.76-7.77(2 \mathrm{H}, \mathrm{m}, \mathrm{HAr}), 7.85\left(2 \mathrm{H}, \mathrm{d},{ }^{3} J_{\mathrm{HH}} 8.0 \mathrm{~Hz}, \mathrm{HAr}\right), 8.70(1 \mathrm{H}, \mathrm{s}$, oxazole proton). ${ }^{13} \mathrm{C}-\mathrm{NMR}\left(100 \mathrm{MHz}, \mathrm{DMSO}-d_{6}\right): \delta(\mathrm{ppm}) 21.60\left(\mathrm{CH}_{3}\right), 116.25\left(\mathrm{~d},{ }^{2} J_{\mathrm{CF}} 24 \mathrm{~Hz}\right), 118.44\left(\mathrm{~d},{ }^{2} J_{\mathrm{CF}} 20\right.$ $\mathrm{Hz}), 125.71,127.69$ (d, ${ }^{3} J_{\mathrm{CF}} 8.0 \mathrm{~Hz}$ ), 128.34, 130.59, 131.43 (d $\left.{ }^{3} J_{\mathrm{CF}} 8.0 \mathrm{~Hz}\right), 135.77,136.92,145.73,150.76$, $152.50(\mathrm{C}=\mathrm{N}), 162.14$ (d, $\left.{ }^{1} \mathrm{~J}_{\mathrm{CF}} 242.0 \mathrm{~Hz}\right)$. Calcd. For $\left(\mathrm{C}_{16} \mathrm{H}_{12} \mathrm{FNO}_{3} \mathrm{~S}\right): \mathrm{C}, 60.55 ; \mathrm{H}, 3.83 ; \mathrm{N}, 4.40 ; \mathrm{S}, 10.12 \%$. Found: C, 60.56; H, 3.81; N, 4.41; S, 10.10\%.

5-(3-Chlorophenyl)-4-tosyloxazole (5e). Colorless crystals, mp 260-262 ${ }^{\circ} \mathrm{C}$ (Yield: $\left.90 \%\right) . \mathrm{IR}(\mathrm{KBr}) \bar{U}_{\max }, \mathrm{cm}^{-1}$; $1609(\mathrm{C}=\mathrm{N})$ 1329, $1149(\mathrm{~S}=\mathrm{O}) .{ }^{1} \mathrm{H}-\mathrm{NMR}\left(400 \mathrm{MHz}, \mathrm{DMSO}-d_{6}\right): \delta(\mathrm{ppm}) 2.40\left(3 \mathrm{H}, \mathrm{S}, \mathrm{CH}_{3}\right), 7.42\left(2 \mathrm{H}, \mathrm{d},{ }^{3} \mathrm{~J}_{\mathrm{HH}}=8.0\right.$ $\mathrm{Hz}, \mathrm{HAr}), 7.44-7.47(3 \mathrm{H}, \mathrm{m}, \mathrm{HAr}), 7.60(1 \mathrm{H}, \mathrm{s}, \mathrm{HAr}), 7.84\left(2 \mathrm{H}, \mathrm{d},{ }^{3}{ }_{\mathrm{HH}} 8.0 \mathrm{~Hz}, \mathrm{HAr}\right), 8.60(1 \mathrm{H}, \mathrm{s}$, oxazole proton). ${ }^{13} \mathrm{C}-\mathrm{NMR}(100 \mathrm{MHz}$, DMSO-d $): \delta(\mathrm{ppm}) 21.26\left(\mathrm{CH}_{3}\right), 124.79125 .97,127.94,128.55,128.74,129.24,129.52$, $132.52,137.04,138.12,143.55,146.15,157.71(\mathrm{C}=\mathrm{N})$ Calcd. for $\left(\mathrm{C}_{16} \mathrm{H}_{12} \mathrm{ClNO}_{3} \mathrm{~S}\right): \mathrm{C}, 57.56 ; \mathrm{H}, 30.64 ; \mathrm{N}, 4.19 ; \mathrm{S}$, 9.60\%. Found: $C, 57.57 ; \mathrm{H}, 30.62 ; \mathrm{N}, 4.20 ; \mathrm{S}, 9.61 \%$.

5-(p-Tolyl)-4-tosyloxazole (5f). Dark brown solid, mp $281-282^{\circ} \mathrm{C}($ Yield: $98 \%)$. IR $(\mathrm{KBr})\left(\overline{\mathrm{U}}_{\max }, \mathrm{cm}^{-1}\right) ; 1597(\mathrm{C}=\mathrm{N})$ 1316, $1146(\mathrm{~S}=\mathrm{O}) .{ }^{1} \mathrm{H}-\mathrm{NMR}\left(400 \mathrm{MHz}, \mathrm{DMSO}-d_{6}\right): \delta(\mathrm{ppm}) 2.40,2.41\left(6 \mathrm{H}, \mathrm{s}, 2 \mathrm{CH}_{3}\right), 7.40\left(2 \mathrm{H}, \mathrm{d},{ }^{3} \mathrm{JHH}^{8} 8.1 \mathrm{~Hz}, \mathrm{HAr}\right.$ ),7.46 (2H, d, $\left.{ }^{3} \mathrm{~J}_{\mathrm{HH}} 8.1 \mathrm{~Hz}, \mathrm{HAr}\right), 7.78-7.83(4 \mathrm{H}, \mathrm{m}, \mathrm{HAr}), 8.84\left(1 \mathrm{H}, \mathrm{s}\right.$, oxazole proton); ${ }^{13} \mathrm{C}-\mathrm{NMR}(100 \mathrm{MHz}$, DMSO- $\left.d_{6}\right) 21.38,21.57\left(2 \mathrm{CH}_{3}\right), 125.67,126.65,128.25,129.04,129.67,130.52,132.11,135.00,137.25$, 138.45, 145.51, 152.07, $152.44(\mathrm{C}=\mathrm{N})$. Calcd. For $\left(\mathrm{C}_{17} \mathrm{H}_{15} \mathrm{NO}_{3} \mathrm{~S}\right)$ : C, 65.15; $\mathrm{H}, 4.82 ; \mathrm{N}, 4.49 ; \mathrm{S}, 10.25 \%$. Found: $\mathrm{C}$, 65.16; H, 4.82; N, 4.47; S, 10.23\%.

5-(m-Tolyl)-4-tosyloxazole (5g). Dark brown solid, mp 270-272 ${ }^{\circ} \mathrm{C}$ (Yield: 98\%). IR (KBr) $\left(\overline{\mathrm{U}}_{\max }, \mathrm{cm}^{-1}\right) ; 1594(\mathrm{C}=\mathrm{N})$ 1313, $1143(\mathrm{~S}=0) .{ }^{1} \mathrm{H}-\mathrm{NMR}\left(400 \mathrm{MHz}, \mathrm{DMSO}-d_{6}\right): \delta(\mathrm{ppm}) 2.40\left(6 \mathrm{H}, \mathrm{S}, 2 \mathrm{CH}_{3}\right), 7.40\left(1 \mathrm{H}, \mathrm{d},{ }^{3} \mathrm{~J}_{\mathrm{HH}} 8.1 \mathrm{~Hz}, \mathrm{HAr}\right), 7.45-$ $7.47(3 \mathrm{H}, \mathrm{m}, \mathrm{HAr}), 7.68(1 \mathrm{H}, \mathrm{s}, \mathrm{HAr}), 7.71\left(1 \mathrm{H}, \mathrm{d},{ }^{3} \mathrm{~J}_{\mathrm{HH}} 8.1 \mathrm{~Hz}, \mathrm{HAr}\right), 7.84\left(2 \mathrm{H}, \mathrm{d},{ }^{3} \mathrm{~J}_{\mathrm{HH}} 8.0 \mathrm{~Hz}, \mathrm{HAr}\right), 8.64(1 \mathrm{H}, \mathrm{s}$, oxazole proton). ${ }^{13} \mathrm{C}-\mathrm{NMR}\left(100 \mathrm{MHz}, \mathrm{DMSO}-d_{6}\right): \delta(\mathrm{ppm}) 21.38,21.57\left(2 \mathrm{CH}_{3}\right), 125.67,126.65,128.25,129.04$, $129.67,130.52,132.11,135.00,137.25,138.45,145.51,152.07,152.44(\mathrm{C}=\mathrm{N})$. Calcd. For $\left(\mathrm{C}_{17} \mathrm{H}_{15} \mathrm{NO}_{3} \mathrm{~S}\right): \mathrm{C}$, 65.14; H, 4.84; N, 4.48; S, 10.20\%. Found: C, 65.16; H, 4.82; N, 4.47; S, 10.23\%.

5-(Methoxymethyl)-4-tosyloxazole (5h). Brown powder, mp 200-202 ${ }^{\circ} \mathrm{C}$ (Yield: 98\%). IR (KBr) $\left(\overline{\mathrm{U}}_{\max }, \mathrm{cm}^{-1}\right)$; $\left.1595(\mathrm{C}=\mathrm{N}) 1334,1152(\mathrm{~S}=\mathrm{O}) .{ }^{1} \mathrm{H}-\mathrm{NMR}(400 \mathrm{MHz} \text { DMSO-d })_{6}\right): \delta(\mathrm{ppm}) 2.41\left(3 \mathrm{H}, \mathrm{s}, \mathrm{CH}_{3}\right), 3.33\left(3 \mathrm{H}, \mathrm{s}, \mathrm{OCH}_{3}\right), 4.82$ $\left(3 \mathrm{H}, \mathrm{s}, \mathrm{OCH}_{2}\right), 7.48\left(2 \mathrm{H}, \mathrm{d},{ }^{3} \mathrm{~J}_{\mathrm{HH}} 8.1 \mathrm{~Hz}, \mathrm{HAr}\right), 7.87\left(2 \mathrm{H}, \mathrm{d},{ }^{3} \mathrm{~J}_{\mathrm{HH}} 8.1 \mathrm{~Hz}, \mathrm{HAr}\right) 8.61\left(1 \mathrm{H}, \mathrm{s}\right.$, oxazole proton). ${ }^{13} \mathrm{C}-\mathrm{NMR}$ $\left(100 \mathrm{MHz}, \mathrm{DMSO}-d_{6}\right): \delta(\mathrm{ppm}) 21.58\left(\mathrm{CH}_{3}\right), 58.59\left(\mathrm{OCH}_{3}\right), 62.60\left(\mathrm{OCH}_{2}\right), 128.23,128.60,130.64,137.58$, 145.71, 152.18, 153.39 (C=N). Calcd. For $\left(\mathrm{C}_{12} \mathrm{H}_{13} \mathrm{NO}_{4} \mathrm{~S}\right): \mathrm{C}, 53.90 ; \mathrm{H}, 4.91 ; \mathrm{N}, 5.26 ; \mathrm{S}, 11.99 \%$. Found: $\mathrm{C}, 53.92 ; \mathrm{H}$, $4.90 ; \mathrm{N}, 5.24 ; \mathrm{S}, 12.00 \%$.

5-(1,2-Dichloro-2-methylpropyl)-4-tosyloxazole (5i). Light brown powder, mp 180-182 ${ }^{\circ} \mathrm{C}$ (Yield: $\left.98 \%\right)$. IR (KBr) $\left(\bar{U}_{\max }, \mathrm{cm}^{-1}\right) ; 1595(\mathrm{C}=\mathrm{N})$ 1326, $1148(\mathrm{~S}=0) .{ }^{1} \mathrm{H}-\mathrm{NMR}\left(400 \mathrm{MHz}, \mathrm{DMSO}-d_{6}\right): \delta(\mathrm{ppm}) 1.21,1.49,(6 \mathrm{H}, \mathrm{s}, 2 \mathrm{CH})$, 2.49, $\left(3 \mathrm{H}, \mathrm{s}, \mathrm{CH}_{3}\right), 4.40(1 \mathrm{H}, \mathrm{s}, \mathrm{CH}), 7.50\left(2 \mathrm{H}, \mathrm{d},{ }^{3} \mathrm{~J}_{\mathrm{HH}} 8.1 \mathrm{~Hz}, \mathrm{HAr}\right), 7.90\left(2 \mathrm{H}, \mathrm{d},{ }^{3} J_{\mathrm{HH}} 8.1 \mathrm{~Hz}, \mathrm{HAr}\right) 8.57(1 \mathrm{H}, \mathrm{s}$, oxazole proton); ${ }^{13} \mathrm{C}-\mathrm{NMR}\left(100 \mathrm{MHz}, \mathrm{DMSO}-d_{6}\right): \delta(\mathrm{ppm})$ 19.41, 21.61, $24.05\left(3 \mathrm{CH}_{3}\right), 55.26,62.64,128.21$, 130.71, 136.90, 138.24, 145.81, 151.30, 152.95(C=N).Calcd. For $\left(\mathrm{C}_{14} \mathrm{H}_{15} \mathrm{Cl}_{2} \mathrm{NO}_{3} \mathrm{~S}\right): \mathrm{C}, 48.29 ; \mathrm{H}, 4.32 ; \mathrm{N}, 4.01 ; \mathrm{S}$, 9.23\%. Found: C, 48.28; H, 4.34; N, 4.02; S, 9.21\%. 


\section{Acknowledgements}

We gratefully acknowledge, the Vail-e-Asr University of Rafsanjan Faculty Research Grant for financial support.

\section{Supplementary Material}

Copies of FT-IR, ${ }^{1} \mathrm{H}$ and ${ }^{13} \mathrm{C}$ NMR spectras for compounds $\mathbf{5 a - I .}$

\section{References}

1. Semenyuta, I.; Kovalishyn, V.; Tanchuk, V.; Pilyo, S.; Zyabrev, V.; Blagodatnyy, V.; Trokhimenko, O.; Brovarets, V.; Metelytsia, L. Comput. Biol. Chem. 2016, 65, 8.

https://doi.org/10.1016/i.compbiolchem.2016.09.012

2. Murade, C. U.; Subramaniam, V.; Otto, C.; Bennink, M. L. Biophys. J. 2009, 97, 835. https://doi.org/10.1016/j.bpj.2009.05.024

3. Eswaran, S.; Adhikari, A. V.; Ajay Kumar, R. Eur. J. Med. Chem. 2010, 45, 957. https://doi.org/10.1016/i.ejmech.2009.11.036

4. Stokes, N. R.; Baker, N.; Bennett, J. M.; Chauhan, P. K.; Collins, I.; Davies, D. T.; Gavade, M.; Kumar, D.; Lancett, P.; Macdonald, R.; Macleod, L.; Mahajan, A.; Mitchell, J. P.; Nayal, N.; Nayal, Y. N.; Pitt, G. R.; Singh, M.; Yadav, A.; Srivastava, A.; Czaplewski, L. G.; Haydon, D. J. Bioorg. Med. Chem. Lett. 2014, $24,353$. https://doi.org/10.1016/j.bmcl.2013.11.002

5. Norman, B. H.; Lee, L. F.; Masferrer,J. L.; Talley, J. J. U.S. Patent, 5380738 A, 1995.

6. Fernandes, E.; Costa, D.; Toste, S. A.; Lima, J. L.; Reis, S. Free Radic. Biol. Med. 2004, 37, 1895. https://doi.org/10.1016/i.freeradbiomed.2004.09.001

7. Lu, X.; Liu, X.; Wan, B.; Franzblau, S. G.; Chen, L.; Zhou, C.; You, Q. Eur. J. Med. Chem. 2012, 49, 164. https://doi.org/10.1016/i.ejmech.2012.01.007

8. Moraski, G. C.; Chang, M.; Villegas- Estrada, A.; Franzblau, S. G.; Mollmann, U.; Miller, M. J. Eur. J. Med. Chem. 2010, 45, 1703. https://doi.org/10.1016/i.ejmech.2009.12.074

9. Rastogi, N.; Abdul, J., Goh, K. S.; Devallois, A.; Philogene, E.; Bourgeois, P. FEMS Immunol. Med. Microbiol. 1998, 20, 267.

https://doi.org/10.1111/j.1574-695X.1998.tb01136.x

10. Sriram, D.; Yogeeswari, P.; Thirumurugan, R.; Pavana, R. K., J. Med. Chem. 2006, 49, 3448. https://doi.org/10.1021/jm060339h

11. Turchi, I. J.; Dewar, M. J. S. Chem. Rev. 1975, 75, 389. https://doi.org/10.1021/cr60296a002

12. Wasserman, H. H.; Gambale, R. J. J. Am. Chem. Soc. 1985, 107, 1423. https://doi.org/10.1021/ja00291a059

13. Maryanoff, B. E. In Heterocyclic Compounds; Turchi, I. J., Ed.; Wiley \& Sons: New York, 1986; Vol. 45, Chapter 5.

14. Kelly, T. R.; Lang, F. J. Org. Chem. 1996, 61, 4623.

https://doi.org/10.1021/jo960433d 
15. Panek, J. S.; Beresis, R. T. J. Org. Chem. 1996, 61, 6496.

https://doi.org/10.1021/jo960532r

16. Wipf, P.; Miller, C. P. J. Org. Chem. 1993, 58, 3604.

https://doi.org/10.1021/jo00066a004

17. Wipf, P.; Lim, S. J. Am. Chem. Soc. 1995, 117, 558.

https://doi.org/10.1021/ja00106a075

18. Bagley, M. C.; Buck, R. T.; Hind, S. L.; Moody, C. J. Perkin Trans 1. 1998, 591.

https://doi.org/10.1039/a704093h

19. Wipf, P.; Graham, T. H. J. Org. Chem. 2001, 66, 3242.

https://doi.org/10.1021/jo005787q

20. Gabriel, S. Ber. Dtsch. Chem. Ges. 1910, 43, 1283.

https://doi.org/10.1002/cber.19100430219

21. Robinson, R. J. Chem. Soc. 1909, 95, 2167.

https://doi.org/10.1039/СT9099502167

22. Haung, W. S.; Zhang, Y. X.; Yaun, C. Y. Synth. Commun. 1996, 26, 1149.

https://doi.org/10.1080/00397919608003722

23. Bossio, R.; Marcaccini, S.; Pepino, R. Heterocycles, 1986, 24, 2003.

https://doi.org/10.3987/R-1986-04-0983

24. Ozaki, Y.; Maeda, S.; Iwasaki, T.; Matsumoto, K.; Odawara, A.; Sasaki, Y.; Morita, T. Chem. Pharm. Bull. 1983, 12, 4417.

https://doi.org/10.1248/cpb.31.4417

25. Ohba, M.; Kubo, H.; Seto, S.; Fujii, T.; Ishibashi, H. Chem. Pharm. Bull. 1998, 46, 860.

https://doi.org/10.1248/cpb.46.860

26. Tang, J.; Varkade, J. G. J. Org. Chem. 1994, 59, 7793.

https://doi.org/10.1021/jo00104a041

27. Sasaki, H.; Kitagawa, T. Chem. Pharm. Bull. 1988, 36, 1593.

https://doi.org/10.1248/cpb.36.1593

28. Brahma, S.; Ray, J. K. J. Heterocycl. Chem. 2008, 45, 311.

https://doi.org/10.1002/jhet.5570450203

29. Tiwari, D. K.; Pogula, J. RSC Adv. 2015, 5, 53111.

https://doi.org/10.1039/C5RA07668D

30. Gouttebaron, R.; Cornelissen, D.; Snyders, R.; Dauchot, J. P.; Wautelet, M.; Hecq, M. JITF. 2000, $30,527$.

31. Li, B.; Buzon, R. A.; Zhang, Z. J. Org. Process Res. Dev. 2007, 11, 951. https://doi.org/10.1021/op700176n

32. Baumann, M.; Baxendale, I. R.; Ley, S. V.; Smith, C. D.; Tranmer, G. K. Org. Lett. 2006, 8, 5231. https://doi.org/10.1021/ol061975c

33. Miller, R. A.; Smith, R. M.; Marcune, B. J. Org. Chem. 2005, 70, 9074.

https://doi.org/10.1021/jo051490m

34. Davies, J. R.; Kane, P. D.; Moody, C. J. Tetrahedron, 2004, 60, 3967.

https://doi.org/10.1016/j.tet.2004.03.037

35. Xia, Q.; Ganem, B. Synthesis 2002, 1969.

36. Ohba, M.; Kubo, H.; Seto, S.; Fujii, T.; Ishibashi, H. Chem. Pharm. Bull. 1998, 46, 860.

https://doi.org/10.1248/cpb.46.860

37. Matsumoto, K.; Suzuki, M.; Yoneda, N.; Miyoshi, M. Synthesis 1976, 805.

38. Possel, O.; Van Leusen, A. Heterocycles 1977, 7, 77. 
https://doi.org/10.3987/S-1977-01-0077

39. van Leusen, A. M.; Hoogenboom, B. E.; Siderius, H. Tetrahedron Lett. 1972, 13, 2369.

https://doi.org/10.1016/S0040-4039(01)85305-3

40. Kulkarni, B. A.; Ganesan, A. Tetrahedron Lett. 1999, 40, 5637.

https://doi.org/10.1016/S0040-4039(99)01050-3

41. Schöllkopf, U.; Gerhart, E. Angew. Chem., Int. Ed. Engl. 1968, 7, 805.

https://doi.org/10.1002/anie.196808051

42. Schöllkopf, U.; Schröder, R. Angew. Chem., Int. Ed. Engl. 1971, 10, 333.

https://doi.org/10.1002/anie.197103331

43. Yeh, V. S. C. Tetrahedron 2004, 60, 11995.

https://doi.org/10.1016/j.tet.2004.10.001

44. Martin, R.; Cuenca, A.; Buchwald, S. L. Org. Lett. 2007, 9, 5521.

https://doi.org/10.1021/ol7024718

45. Herrera, A.; Martinez- Alvarez, R.; Ramiro, P.; Molero, D.; Almy, J. J. Org. Chem. 2006, 71, 3026. https://doi.org/10.1021/jo052619v

46. Davies, J. R.; Kane, P. D.; Moody, C. J. Tetrahedron 2004, 60, 3967. https://doi.org/10.1016/i.tet.2004.03.037

47. Shi, B.; Blake, A. J.; Campbell, I. B.; Judkins, B. D.; Moody, C. J. Chem. Comm, 2009, 22, 3291. https://doi.org/10.1039/b903878g

48. Hu, Y.; Yi, R.; Wang, C.; Xin, X.; Wu, F.; Wan, B. J. Org. Chem. 2014, 79, 3052.

https://doi.org/10.1021/jo5001719

49. Ozaki, Y.; Maeda, S.; Iwasaki, T.; Matsumoto, K.; Odawara, A.; Sasaki, Y.; Morita, T. Chem. Pharm. Bull. 1983, 31, 4417.

https://doi.org/10.1248/cpb.31.4417

50. Marcus, Bauman, M.; Baxendale, I.R.; Ley, S.V.; Smith, C.D.; Tranmer, G.K. Org. Lett. 2006, 8, 5231. https://doi.org/10.1021/ol061975c 